\title{
THE NUMERICAL ANALYSIS OF IMPLICIT RUNGE-KUTTA METHODS FOR A CERTAIN NONLINEAR INTEGRO-DIFFERENTIAL EQUATION
}

\author{
YUAN WEI ${ }^{*}$ AND TANG TAO ${ }^{* *}$
}

ABSTRACT. In this paper and in an earlier 1987 paper, the mathematical theory and numerical methods for the nonlinear integro-differential equation

$$
\begin{aligned}
& u^{\prime}(t)+p(t) u(t)+\int_{0}^{t} k(t, s) u(t-s) u(s) d s=q(t), \quad 0 \leq t \leq T, \\
& u(0)=u_{0}
\end{aligned}
$$

are considered. Equations of this type occur as model equations for describing turbulent diffusion. Previously, the existence and uniqueness properties of the solutions of the model equation were solved completely, and a class of implicit Runge-Kutta methods with $m$ stages for the approximate solution of the model equation was introduced. In this paper, we give a further numerical analysis of these methods. It is proved that the implicit Runge-Kutta methods with $m$ stages are of optimal approximation order $p=2 m$. Some computational examples are given.

\section{INTRODUCTION}

We consider the approximate solution of the initial value problem for a nonlinear integro-differential equation

$$
\begin{aligned}
& u^{\prime}(t)+p(t) u(t)+\int_{0}^{t} k(t, s) u(t-s) u(s) d s=q(t), \quad t \in I:=[0, T], \\
& u(0)=u_{0},
\end{aligned}
$$

where $p(t), q(t)$ and $k(t, s)$ are continuous functions in $I$ and $S:=\{(t, s) \mid$ $0 \leq s \leq t \leq T\}$, respectively. Equations of this type arise as model equations for describing turbulent diffusion problems (see [18] and [22]). In [8], Chang and Day investigated the existence and uniqueness properties of the solution of (1.1) under certain conditions and presented a linear multistep method for obtaining an approximate solution with order of (local) convergence $p=4$. In [21] we have solved completely the existence and uniqueness properties of the

Received July 16, 1986; revised September 23, 1987, March 29, 1988 and October 14, 1988.

1980 Mathematics Subject Classification (1985 Revision). Primary 65R99, 45L10; Secondary $45 \mathrm{~J} 05$.

* Current address: Center for Applied Mathematics, Cornell University, Ithaca, NY 14853, U.S.A. England.

" Current address: Department of Applied Mathematics, University of Leeds, Leeds LS2 9JT, 
solution of (1.1) and introduced a class of implicit Runge-Kutta methods with $m$ stages for obtaining an approximate solution of (1.1).

There has been a great deal of recent work in the numerical analysis of the implicit Runge-Kutta methods for Volterra integro-differential equations. Nonoptimal methods have been discussed by many authors: in [13] and [14], Lubich presents a general theory conterning the structure of the order conditions of Runge-Kutta methods for the nonlinear Volterra integro-differential equations of the form

$$
\begin{aligned}
& u^{\prime}(t)=F\left(t, u(t), \int_{0}^{t} k(t, s, u(s)) d s\right), \quad t \in I, \\
& u(0)=u_{0},
\end{aligned}
$$

and he lists a number of special explicit methods; Makroglou [15] investigates related methods called block-by-block methods (compare also Mocarsky [17], Brunner [3], Brunner and Lambert [5, pp. 84-87], and Feldstein and Sopka [12]). In 1984, Brunner presented optimal methods and their numerical analysis for a special form of (1.2),

$$
\begin{aligned}
& u^{\prime}(t)=f(t, u(t))+\int_{0}^{t} k(t, s, u(s)) d s, \quad t \in I, \\
& u(0)=u_{0},
\end{aligned}
$$

by means of collocation techniques in certain polynomial spline spaces [4]. In [1], Aguilar and Brunner present an optimal method for a class of secondorder Volterra integro-differential equations. The book of Brunner and van der Houwen [6] reviews the state of the numerical solution of Volterra equations. However, a complete convergence theory, including local superconvergence results and the discretization of the collocation equations, has now been obtained by Brunner [4]. His paper has in many ways been a model for the present work.

Volterra integro-differential equations arising in mathematical models of certain biological and physical phenomena are often of a "nonstandard" form, different from (1.2) (see, e.g., Volterra [23], Saaty [20, pp. 301-345], Prosperetti [19], McKee [16], Elliott and McKee [11], and Dixon [10]). The collocation methods described by Brunner [4] (compare also Brunner and van der Houwen [6]) are readily extended to such equations. However, in some cases the convergence analysis is still lacking. It is hoped that the present work will help in dealing with such nonstandard forms of the Volterra integro-differential equation, using collocation methods.

The numerical methods to be analyzed can be obtained by fully discretized collocation in the piecewise polynomial space

$$
S_{m}^{(0)}\left(Z_{N}\right)=\left\{y\left|y \in C(I), y_{n}=y\right|_{\sigma_{n}} \in \pi_{m}, n=0, \ldots, N-1\right\} .
$$

Here, $N \geq 1$ and $m \geq 1$ are positive integers, $\pi_{m}$ denotes the space of real polynomials of degree not exceeding $m, h=T / N, t_{0}=0, t_{n}=t_{0}+n \cdot h$ $(n=0,1, \ldots, N), Z_{N}:=\left\{t_{n} \mid n=1, \ldots, N-1\right\}, \bar{Z}_{N}=Z_{N} \cup T$, and $\sigma_{n}:=$ $\left[t_{n}, t_{n+1}\right](0 \leq n \leq N-1)$.

Let $X(N)=\bigcup_{n=0}^{N-1} X_{n}$, where

$$
X_{n}=\left\{t_{n}+c_{i} h \mid 0 \leq c_{1}<\cdots<c_{m} \leq 1\right\}, \quad n=0, \ldots, N-1,
$$


denote the set of collocation points at which the approximate solution $y(t) \in$ $S_{m}^{(0)}\left(Z_{N}\right)$ is to satisfy the equation (1.1). Thus, the approximate solution $y(t)$ is determined recursively by

$$
\begin{aligned}
y_{n}^{\prime}\left(t_{n}\right. & \left.+c_{i} h\right)+p\left(t_{n}+c_{i} h\right) y_{n}\left(t_{n}+c_{i} h\right) \\
& +\int_{t_{n}}^{t_{n}+c_{i} h} k\left(t_{n}+c_{i} h, s\right) y_{n}(s) y_{0}\left(t_{n}+c_{i} h-s\right) d s \\
& +\sum_{k=0}^{n-1} \int_{t_{k}}^{t_{k}+c_{i} h} k\left(t_{n}+c_{i} h, s\right) y_{k}(s) y_{n-k}\left(t_{n}+c_{i} h-s\right) d s \\
& +\sum_{k=0}^{n-1} \int_{t_{k}+c_{i} h}^{t_{k+1}} k\left(t_{n}+c_{i} h, s\right) y_{k}(s) y_{n-k-1}\left(t_{n}+c_{i} h-s\right) d s \\
= & q\left(t_{n}+c_{i} h\right), \quad i=1, \ldots, m, n=0,1, \ldots, N-1 .
\end{aligned}
$$

Since $y(t) \in C(I)$, we have

$$
y_{n}\left(t_{n}\right)=y_{n-1}\left(t_{n}\right)
$$

In this paper we show that the attainable order of collocation approximation for the nonlinear equation $(1.1)$ is $p=2 \mathrm{~m}$.

\section{The ATTAINABLE ORDER OF COLLOCATION APPROXIMATION}

In this section we shall present the attainable order of the collocation approximation (1.6) and (1.7).

It is easy to see that for each $y_{n}(t), n=0, \ldots, N-1$, Eq. (1.6) is linear except for $y_{0}(t)$. We first consider the case of $n=0$. We shall use the wellknown contraction principle stated in the following lemma:

Lemma 2.1. Let $(X, d)$ be a complete metric space and

$$
B\left(x_{0}, r\right)=\left\{x \in X \mid d\left(x, x_{0}\right)<r\right\}
$$

a sphere in $X$ with radius $r$, centered at $x_{0}$. Suppose that $T: B\left(x_{0}, r\right) \rightarrow X$ is a contraction mapping satisfying

$$
d(T x, T y) \leq L \cdot d(x, y), \quad 0<L<1, \forall x, y \in B\left(x_{0}, r\right) .
$$

If $d\left(T x_{0}, x_{0}\right)<(1-L) \cdot r$, then there exists a unique fixed point of $T$ in $B\left(x_{0}, r\right)$.

From (1.6) with $n=0$, we get

$$
\begin{gathered}
y_{0}^{\prime}\left(c_{i} h\right)+p\left(c_{i} h\right) y_{0}\left(c_{i} h\right)+h \int_{0}^{c_{1}} k\left(c_{i} h, \tau h\right) y_{0}(\tau h) y_{0}\left(\left(c_{i}-\tau\right) h\right) d \tau \\
=q\left(c_{i} h\right), \quad i=1, \ldots, m .
\end{gathered}
$$

From the definition of $S_{m}^{(0)}\left(Z_{N}\right)$, and $v_{0}(0)=u_{0}$, we have

$$
y_{0}(\tau h)=u_{0}+\sum_{j=1}^{m} \psi_{j} \cdot \tau^{j}, \quad \tau \in(0,1) \text {, }
$$


hence

$$
y_{0}^{\prime}(\tau h)=h^{-1} \cdot \sum_{j=1}^{m} j \cdot \psi_{j} \tau^{j-1}, \quad \tau \in(0,1) .
$$

Substituting (2.2) and (2.3) into (2.1), and following the lines of proof of Theorem 2.1 in [4], we can show that there exists $h_{1}>0$ such that for $h \in\left(0, h_{1}\right)$ the $\left\{\psi_{j}\right\}$ in $(2.2),(2.3)$ satisfy

$$
\psi_{i}=h g_{i}-h^{2} \sum_{j, l=1}^{m} c_{j l}^{(i)} \psi_{j} \psi_{l}, \quad i=1, \ldots, m,
$$

where $g_{i}$ and $c_{j l}^{(i)}$ are bounded by constants which do not depend on $h$.

Let $\psi=\left\{\psi_{1}, \ldots, \psi_{m}\right\}^{t}$. Define the operator $T: R^{m} \rightarrow R^{m}$ as follows:

$$
(T \psi)_{i}=h g_{i}-h^{2} \sum_{j, l=1}^{m} c_{j l}^{(i)} \cdot \psi_{j} \cdot \psi_{l}, \quad i=1, \ldots, m .
$$

We consider the set $B(0, r)=\left\{\psi \in R^{m} \mid\|\psi\|_{1}<r\right\}$, where $r>0$ and

$$
\|\psi\|_{1}=\sum_{i=1}^{m}\left|\psi_{i}\right| \text {. }
$$

$T$ is a continuous operator from $B(0, r)$ into $R^{m}$, and for all $\lambda, \psi \in B(0, r)$ we have

$$
\begin{aligned}
\|T \lambda-T \psi\|_{1} & =\sum_{i=1}^{m}\left|(T \lambda)_{i}-(T \psi)_{i}\right| \\
& =h^{2} \cdot \sum_{i=1}^{m}\left|\sum_{j, l=1}^{m} c_{j l}^{(i)}\left(\lambda_{l} \lambda_{j}-\psi_{l} \psi_{j}\right)\right| \\
& \leq h^{2} \sum_{i=1}^{m}\left\{\sum_{j, l=1}^{m}\left(\left|c_{j l}^{(i)}\right| \cdot\left|\lambda_{l}\right|+\left|c_{l j}^{(i)}\right| \cdot\left|\psi_{l}\right|\right) \cdot\left|\lambda_{j}-\psi_{j}\right|\right\} \\
& \leq h^{2} m \cdot \max _{i, j, l}\left|c_{j l}^{(i)}\right| \cdot\left(\|\lambda\|_{1}+\|\psi\|_{1}\right) \cdot\|\lambda-\psi\|_{1} \\
& \leq L \cdot\|\lambda-\psi\|_{1},
\end{aligned}
$$

where $L=2 h^{2} \cdot r \cdot m \cdot \max _{i, j, l}\left|c_{j l}^{(i)}\right|$.

On the other hand, let $x_{0}=0$; then

$$
\left\|T x_{0}-x_{0}\right\|_{1}=h \sum_{i=1}^{m}\left|g_{i}\right| \leq m h \cdot \max _{i}\left|g_{i}\right| \text {. }
$$

Let $F_{1}=m \cdot \max \left|g_{i}\right|$ and $F_{2}=2 \cdot m \cdot \max \left|c_{j l}^{(i)}\right|$. We seek to determine $h$ sufficiently small so as to have $L<1$ and $\left\|T x_{0}-x_{0}\right\|_{1} \leq(1-L) \cdot r$. Since $F_{1}$ and $F_{2}$ are bounded when $h \in\left(0, h_{1}\right)$, if we let

$$
h_{2}=\left(4 \cdot F_{1} \cdot F_{2}\right)^{-1 / 3}
$$


(so that $h \in\left(0, h_{2}\right)$ implies $2 \cdot F_{1} \cdot h \leq\left(2 \cdot F_{2} \cdot h^{2}\right)^{-1}$ ), then it is easy to see that when $h \in(0, \tilde{h})$, where $\tilde{h}=\min \left\{h_{1}, h_{2}\right\}$, and if $r>0$ satisfies

$$
2 \cdot F_{1} \cdot \tilde{h} \leq r \leq\left(2 \cdot F_{2} \cdot \tilde{h}^{2}\right)^{-1},
$$

then

$$
L=h^{2} \cdot F_{2} \cdot r \leq 1 / 2
$$

and

$$
\left\|T x_{0}-x_{0}\right\|_{1} \leq h \cdot F_{1} \leq \frac{1}{2} r \leq(1-L) \cdot r .
$$

Therefore, from Lemma 2.1, we obtain that for $r>0$ satisfying (2.8) there is a unique fixed point $\psi^{*}$ of $T$ in $B(0, r)$ when $h \in(0, \tilde{h})$, i.e.,

$$
\psi^{*}=T \psi^{*} \text {. }
$$

That is, if we compute the $\left\{\psi_{j}\right\}$ by means of the iteration

$$
\left\{\begin{array}{l}
\psi^{(k+1)}=T \psi^{(k)}, \quad k=0,1,2, \ldots, \\
\text { with } \psi^{(0)} \in B(0, r),
\end{array}\right.
$$

then for any $h \in(0, \tilde{h})$ we obtain a sequence $\psi^{(k)} \in B(0, r)$ which converges to $\psi^{*} \in B(0, r)$, the unique solution of $(2.4)$. Moreover, there exists a constant $B$ such that

$$
\left\|y_{0}(t)\right\|_{\infty} \leq B \quad \forall h \in(0, \tilde{h})
$$

Thus we have

Theorem 2.1. Let $p(t), q(t)$ and $k(t, s)$ be continuous functions in their respective domains. If we compute $y_{0}(t)$ by (2.2) and (2.10) (i.e., using the simple iteration method), there exists $\tilde{h}>0$ such that for any $r>0$ satisfying (2.8), and for arbitrary starting vector $\psi^{(0)} \in B(0, r)$, the iterates $\psi^{(k)}$ converge to the unique solution of $(2.9)$ in $B(0, r)$ for any $h \in(0, \tilde{h})$. Furthermore, $y_{0}(t)$ is bounded, i.e., $\left\|y_{0}\right\|_{\infty} \leq B$, where $B$ is a constant which is independent of $h$.

Using Theorem 2.1, we shall prove the following convergence theorem.

Theorem 2.2. Suppose that $p(t), q(t) \in C^{m}(I)$ and $\partial^{j} k(t, s) / \partial t^{j-r} \partial s^{r}, 0 \leq$ $r \leq j \leq m$, are continuous functions in $I \times I$. Then there exists $\bar{h}>0$ such that for any choice of the collocation parameters $\left\{c_{i}\right\}$ with $0 \leq c_{1}<\cdots<c_{m} \leq 1$, the error $e(t)=u(t)-y(t)$ satisfies

$$
\left\{\begin{aligned}
&\|e\|_{\infty}=\max \{|e(t)| \mid t \in I\}=O\left(h^{m}\right), \\
&\left\|e^{\prime}\right\|_{\infty}=\sup \left\{\mid e_{n}^{\prime}(t) \| t \in \sigma_{n},\right.n=0,1, \ldots, N-1\}=O\left(h^{m}\right) \\
& \text { as } h \rightarrow 0_{+}, N \cdot h=T .
\end{aligned}\right.
$$

Proof. Set $t=t_{n}+c_{i} h$ in (1.1) and note (1.6). Then

$$
\begin{aligned}
& e_{n}^{\prime}\left(t_{n}+c_{i} h\right)+p\left(t_{n}+c_{i} h\right) e_{n}\left(t_{n}+c_{i} h\right) \\
& \quad+\int_{0}^{t_{n}+c_{i} h} k\left(t_{n}+c_{i} h, s\right)\left[u(s) u\left(t_{n}+c_{i} h-s\right)-y(s) y\left(t_{n}+c_{i} h-s\right)\right] d s \\
& \quad=0, \quad i=1, \ldots, m,
\end{aligned}
$$


and it is not difficult to obtain

$$
\begin{array}{rl}
\int_{0}^{t_{n}+c_{i} h} & k\left(t_{n}+c_{i} h, s\right)\left[u(s) u\left(t_{n}+c_{i} h-s\right)-y(s) y\left(t_{n}+c_{i} h-s\right)\right] d s \\
= & \int_{t_{n}}^{t_{n}+c_{i} h} \hat{k}\left(t_{n}+c_{i} h, s\right) e_{n}(s) d s+\sum_{k=0}^{n-1} \int_{t_{k}}^{t_{k+1}} \tilde{k}\left(t_{n}+c_{i} h, s\right) e_{k}(s) d s \\
= & h \int_{0}^{c_{i}} \hat{k}\left(t_{n}+c_{i} h, t_{n}+\tau h\right) e_{n}\left(t_{n}+\tau h\right) d \tau \\
& +h \sum_{k=0}^{n-1} \int_{0}^{1} \tilde{k}\left(t_{n}+c_{i} h, t_{k}+\tau h\right) e_{k}\left(t_{k}+\tau h\right) d \tau,
\end{array}
$$

where

$$
\begin{aligned}
\hat{k}\left(t_{n}+\right. & \left.c_{i} h, s\right) \\
& =\left\{\begin{array}{r}
k\left(c_{i} h, s\right) u\left(c_{i} h-s\right)+k\left(c_{i} h, c_{i} h-s\right) y_{0}\left(c_{i} h-s\right) \quad \text { if } n=0, \\
{\left[k\left(t_{n}+c_{i} h, t_{n}+c_{i} h-s\right)+k\left(t_{n}+c_{i} h, s\right)\right] y_{0}\left(t_{n}+c_{i} h-s\right)} \\
\text { if } n \geq 1, t_{n} \leq s \leq t_{n}+c_{i} h,
\end{array}\right.
\end{aligned}
$$

and

$$
\begin{aligned}
\tilde{k}\left(t_{n}+\right. & \left.c_{i} h, s\right) \\
& =\left\{\begin{array}{r}
k\left(t_{n}+c_{i} h, s\right) u\left(t_{n}+c_{i} h-s\right) \\
+k\left(t_{n}+c_{i} h, t_{n}+c_{i} h-s\right) y\left(t_{n}+c_{i} h-s\right), \\
{\left[k\left(t_{n}+c_{i} h, s\right)+k\left(t_{n}+c_{i} h, t_{n}+c_{i} h-s\right)\right] u\left(t_{n}+c_{i} h-s\right),} \\
0 \leq s \leq t_{n} h .
\end{array}\right.
\end{aligned}
$$

Therefore,

$$
\begin{aligned}
& e_{n}^{\prime}\left(t_{n}+c_{i} h\right)+p\left(t_{n}+c_{i} h\right) e_{n}\left(t_{n}+c_{i} h\right) \\
& +h \int_{0}^{c_{1}} \hat{k}\left(t_{n}+c_{i} h, t_{n}+\tau h\right) e_{n}\left(t_{n}+\tau h\right) d \tau \\
& +h \sum_{k=0}^{n-1} \int_{0}^{1} \tilde{k}\left(t_{n}+c_{i} h, t_{k}+\tau h\right) e_{k}\left(t_{k}+\tau h\right) d \tau=0, \\
& \quad i=1, \ldots, m,
\end{aligned}
$$

where $e_{n}=\left.e\right|_{\sigma_{n}}$. From (2.15), (2.16) and (2.17), we can easily see that $\hat{k}\left(t_{n}+c_{i} h, t_{n}+\tau h\right)$ depends only on the functions $k(t, s), u(t)$ and $y_{0}(t)$, and $\tilde{k}\left(t_{n}+c_{i} h, t_{k}+\tau h\right)(k=0, \ldots, n-1)$ depends only on the functions $k(t, s)$, $u(t), y_{n-k}(t) \quad(k=1, \ldots, n-1)$ and $y_{n-k-1}(t) \quad(k=0, \ldots, n-1)$. Therefore, if the functions $y_{k}(t)(k=0, \ldots, n-1)$ are bounded, it will be obvious that $\hat{k}\left(t_{n}+c_{i} h, t_{k}+\tau h\right)$ and $\tilde{k}\left(t_{n}+c_{i} h, t_{k}+\tau h\right)$ are bounded. In this case, using the same method as in the proof of Theorem 2.1 of [4], one easily gets $\left|e_{n}\left(t_{n}+\tau h\right)\right|=O\left(h^{m}\right)$ and $\left|e_{n}^{\prime}\left(t_{n}+\tau h\right)\right|=O\left(h^{\prime n}\right) \quad(0<\tau<1)$, provided $h$ is sufficiently small. 
Now we prove inductively that there are constants $D_{1}, D_{2}$ independent of $h, N$, and $n$, and $\bar{h}>0$, such that

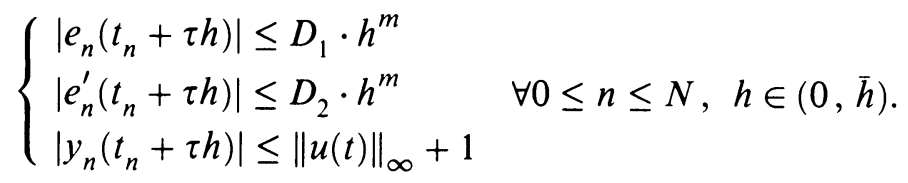

(1) When $n=0$, then (2.17) can be written as

$$
e_{0}^{\prime}\left(c_{i} h\right)+p\left(c_{i} h\right) e_{0}\left(c_{i} h\right)+h \int_{0}^{c_{i}} \hat{k}\left(c_{i} h, \tau h\right) e_{0}(\tau h) d \tau=0, \quad i=1, \ldots, m .
$$

From Theorem 2.1 we have $\left|y_{0}(\tau h)\right| \leq B$. Therefore, using the same method as in the proof of Theorem 2.1 of [4], we have $\left|e_{0}(\tau h)\right|=O\left(h^{m}\right)$ and $\left|e_{0}^{\prime}(\tau h)\right|=$ $O\left(h^{m}\right)$. Thus, by the definition of $e_{n}(t)$, when $h$ is sufficiently small we have $\left|y_{0}(\tau h)\right| \leq\|u(t)\|_{\infty}+1$.

(2) We now assume that the results of (2.18) hold when $0 \leq n \leq M-1$, where $M \geq 1$ is a positive integer. Then

$$
\begin{aligned}
\left|\hat{k}\left(t_{n}+c_{i} h, t_{k}+\tau h\right)\right| \leq 2\|k(t, s)\|_{\infty}\left(\|u(t)\|_{\infty}+1\right), & \left|\tilde{k}\left(t_{n}+c_{i} h, t_{k}+\tau h\right)\right| \leq 2\|k(t, s)\|_{\infty}\left(\|u(t)\|_{\infty}+1\right), \\
& \quad i=1, \ldots, m ; k=0, \ldots, M-1 .
\end{aligned}
$$

Using the same method as in [4], we can show the existence of constants $D_{1}$, $D_{2}$, and $\bar{h}>0$, such that the results of (2.18) hold for $n=M$. This proves Theorem 2.2.

While every choice of the collocation parameters $\left\{c_{i}\right\}$ guarantees the same global convergence rate $p=m$, there exists a special set of these parameters for which one obtains a higher order of convergence at the nodes of the approximating piecewise polynomials. This is made precise in

Theorem 2.3. Suppose that $p(t), q(t) \in C^{2 m-\nu}(\bar{I}), k(t, s) \in C^{2 m-\nu}(\bar{I} \times \bar{I})$, where $\nu \in\{0,1,2\}$.

(a) If $\nu=0$, and if $y \in S_{m}^{(0)}\left(Z_{N}\right)$ denotes the collocation approximation determined by (1.6), where the collocation parameters are the zeros of $\pi_{m}^{(0,1)}(2 s-1)$ (Gauss points on $\left.(0,1)\right)$, then

$$
e\left(t_{n}\right)=O\left(h^{2 m}\right), \quad t_{n} \in \bar{Z}_{N} \text { as } h \rightarrow 0_{+}, \quad N h=T .
$$

(b) If $\nu=1$, and if $y \in S_{m}^{(0)}\left(Z_{N}\right)$ denotes the collocation approximation determined by (1.6), where the collocation parameters are the zeros of $s \cdot \pi_{m-1}^{(0,1)}(2 s-1)$ or of $(s-1) \cdot \pi_{m-1}^{(0,1)}(2 s-1)$ (Radau points on $[0,1)$ and $(0,1]$, respectively), then

$$
e\left(t_{n}\right)=O\left(h^{2 m-1}\right), \quad t_{n} \in \bar{Z}_{N} \text { as } h \rightarrow 0_{+}, \quad N h=T .
$$

(c) If $\nu=2$, and if $y \in S_{m}^{(0)}\left(Z_{N}\right)$ denotes the collocation approximation determined by (1.6), where the collocation parameters are the zeros of $(s-1) s$. $\pi_{m-2}^{(0,1)}(2 s-1)$ (Lobatto points for $\left.[0,1]\right)$, then

$$
e\left(t_{n}\right)=O\left(h^{2 m-2}\right), \quad t_{n} \in \bar{Z}_{N} \text { as } h \rightarrow 0_{+}, \quad N h=T .
$$


Proof. According to (1.6) the collocation solution $y \in S_{m}^{(0)}\left(Z_{N}\right)$ satisfies

$$
y^{\prime}(t)+p(t) y(t)+\int_{0}^{t} k(t, s) y(s) y(t-s) d s=q(t)-\delta(t), \quad t \in I,
$$

where the defect $\delta(t)$ vanishes at $t=0$ and on the set $X(N)$ of collocation points.

Subtraction of (2.22) from (1.1) leads to

$$
\left\{\begin{array}{l}
e^{\prime}(t)+p(t) e(t)+\int_{0}^{t} G(t, s) e(s) d s \\
\quad=\delta(t)+\int_{0}^{t} k(t, s) e(s) e(t-s) d s, \quad t \in I, \\
e(0)=0,
\end{array}\right.
$$

where $G(t, s)=(k(t, s)+k(t, t-s)) u(t-s)$.

The integro-differential equation (2.23) can be viewed as a perturbation of the linear equation

$$
e^{\prime}(t)+p(t) e(t)+\int_{0}^{t} G(t, s) e(s) d s=\delta(t)
$$

with perturbation term $\psi(t):=\int_{0}^{t} k(t, s) e(s) e(t-s) d s$.

For $t=t_{n}, n=0, \ldots, N-1$, we may write

$$
\begin{aligned}
e\left(t_{n}\right)= & R\left(t_{n}, 0\right) e(0)+\int_{0}^{t_{n}} R\left(t_{n}, s\right)\{\delta(s)+\psi(s)\} d s \\
= & h \sum_{k=0}^{n-1} \int_{0}^{1} R\left(t_{n}, t_{k}+\tau h\right) \delta\left(t_{k}+\tau h\right) d \tau \\
& +\int_{0}^{t_{n}} R\left(t_{n}, s\right)\left[\int_{0}^{s} k(s, \tau) e(\tau) e(s-\tau) d \tau\right] d s,
\end{aligned}
$$

where $R(t, s)$ denotes the resolvent kernel associated with the linear equation (2.24). By Theorem 2.2 we know that $\|e\|_{\infty} \leq C h^{m}$. On the other hand (cf. [7, pp. 93-98] or [6]), $R(t, s)$ is a bounded function for $0 \leq s \leq t \leq T$. It follows that

$$
\int_{0}^{t_{n}} R\left(t_{n}, s\right)\left[\int_{0}^{s} k(s, \tau) e(\tau) e(s-\tau) d \tau\right] d s \leq Q h^{2 m}
$$

where the constant $Q$ depends only on the functions $p(t), q(t)$ and $k(t, s)$. Therefore, (2.25) implies

$$
\begin{aligned}
e\left(t_{n}\right) & \leq h\left|\sum_{k=0}^{n-1} \int_{0}^{1} R\left(t_{n}, t_{k}+\tau h\right) \delta\left(t_{k}+\tau h\right) d \tau\right|+Q_{2} h^{2 m} \\
& \leq h \sum_{k=0}^{n-1}\left\{\left|\sum_{j=1}^{m} w_{j} R\left(t_{n}, t_{k}+c_{j} h\right) \delta\left(t_{k}+c_{j} h\right)+E_{n k}\right|\right\}+Q_{2} h^{2 m} \\
& \leq h \sum_{k=0}^{n-1}\left|E_{n k}\right|+Q h^{2 m}
\end{aligned}
$$


where $E_{n k}$ denotes the error term associated with the quadrature formula based on the abscissas $\left\{t_{k}+c_{j} h\right\}$ and the weights $\left\{w_{j}\right\}, j=1, \ldots, m$. Now the arguments used in the proof of Theorem 2.2 of [4] complete the proof.

\section{THE DISCRETIZED COLLOCATION EQUATION: IMPLICIT RUNGE-KUTTA METHODS AND NUMERICAL EXAMPLES}

In most cases the integrals occurring in the collocation equation (1.6) cannot be evaluated analytically and must be approximated by suitable quadrature formulae. This means that, instead of $y$, we compute an approximation $\hat{y} \in S_{m}^{(0)}\left(Z_{N}\right)$ from a perturbed collocation equation

$$
\begin{aligned}
& \hat{y}_{n}^{\prime}\left(t_{n}+c_{i} h\right)+p\left(t_{n}+c_{i} h\right) \hat{y}_{n}\left(t_{n}+c_{i} h\right) \\
& +h \sum_{j=1}^{m} w_{i j} k\left(t_{n}+c_{i} h, t_{n}+c_{i} c_{j} h\right) \hat{y}_{n}\left(t_{n}+c_{i} c_{j} h\right) \hat{y}_{0}\left(\left(c_{i}-c_{i} c_{j}\right) h\right) \\
& +h \sum_{k=0}^{n-1} \sum_{j=1}^{i} w_{j} k\left(t_{n}+c_{i} h, t_{k}+c_{j} h\right) \hat{y}_{k}\left(t_{k}+c_{j} h\right) \hat{y}_{n-k}\left(t_{n-k}+\left(c_{i}-c_{j}\right) h\right) \\
& +h \sum_{k=0}^{n-1} \sum_{j=i+1}^{m} w_{j} k\left(t_{n}+c_{i} h, t_{k}+c_{j} h\right) \hat{y}_{k}\left(t_{k}+c_{j} h\right) \\
& \quad \times \hat{y}_{n-k-1}\left(t_{n-k-1}+\left(1+c_{i}-c_{j}\right) h\right) \\
& =q\left(t_{n}+c_{i} h\right), \quad i=1,2, \ldots, m ; n=0,1, \ldots, N-1 .
\end{aligned}
$$

For (3.1) the same results as in Theorem 3.1 and Corollary 3.1 of [4] hold.

Before presenting numerical examples, we rewrite the discretized collocation equation (3.1) in a form which is convenient for numerical computation and which exhibits more clearly the fact that (3.1) defines a class of implicit RungeKutta methods for the solution of Eq. (1.1). Let $Y_{j}^{(n)}=\hat{y}_{n}^{\prime}\left(t_{n}+c_{i} h\right)$ (where $\left.\hat{y}_{n}^{\prime} \in \pi_{m-1}\right)$ and set

$$
\beta_{j}(x) \prod_{r=1: r \neq j}^{m} \frac{x-c_{r}}{c_{j}-c_{r}}, \quad j=1, \ldots, m .
$$

Since $\hat{y}_{n}^{\prime}\left(t_{n}+\tau h\right)=\sum_{j=1}^{m} \beta_{j}(\tau) \cdot Y_{j}^{(n)}$, we have

$$
\hat{y}_{n}\left(t_{n}+\tau h\right)=\hat{y}_{n}+h \sum_{j=1}^{m} \alpha_{j}(\tau) \cdot Y_{j}^{(n)},
$$

where we have set $\hat{y}_{n}=\hat{y}_{n}\left(t_{n}\right)\left(=\hat{y}_{n-1}\left(t_{n}\right)\right)$ and

$$
\alpha_{j}(\tau)=\int_{0}^{\tau} \beta_{j}(v) d v, \quad j=1, \ldots, m .
$$


Thus, the fully discretized collocation equations can be written in the form: for $n=0$,

$$
\begin{aligned}
Y_{i}^{(0)}+ & \sum_{j=1}^{m}\left[h p\left(c_{i} h\right) \alpha_{j}\left(c_{i}\right)+\hat{y}_{0} h^{2}\right. \\
& \cdot\left(\sum_{r=1}^{m} w_{i r} k\left(c_{i} h, c_{i} c_{r} h\right)\left(\alpha_{j}\left(c_{i} c_{r}\right)+\alpha_{j}\left(c_{i}-c_{i} c_{r}\right)\right)\right] Y_{j}^{(0)} \\
& +h^{3} \sum_{k=1}^{m} \sum_{j=1}^{m}\left[\sum_{r=1}^{m} w_{i r} k\left(c_{i} h, c_{i} c_{r} h\right) \alpha_{j}\left(c_{i} c_{r}\right) \alpha_{k}\left(c_{i}-c_{i} c_{r}\right)\right] Y_{i}^{(0)} Y_{k}^{(0)} \\
= & q\left(c_{i} h\right)-\hat{y}_{0} p\left(c_{i} h\right)-h \hat{y}_{0}^{2} \sum_{j=1}^{m} w_{i j} k\left(c_{i} h, c_{i} c_{j} h\right), \quad i=1, \ldots, m,
\end{aligned}
$$

where $\hat{y}_{0}=u_{0}$, and for $n \geq 1$,

$$
\begin{aligned}
Y_{i}^{(n)}+\sum_{j=1}^{m} A_{i j} \cdot Y_{j}^{(n)} & =-B_{i}^{(n)} \cdot \hat{y}_{n}+C_{i}^{(n)}, \\
i & =1, \ldots, m ; n=1, \ldots, N-1,
\end{aligned}
$$

where

$$
\begin{aligned}
& A_{i j}^{(n)}= h p\left(t_{n}+c_{i} h\right) \alpha_{j}\left(c_{i}\right) \\
&+h^{2} \sum_{r=1}^{m} w_{i r} k\left(t_{n}+c_{i} h, t_{n}+c_{i} c_{r} h\right) \alpha_{j}\left(c_{i} c_{r}\right)\left(\hat{y}_{0}+h \sum_{k=1}^{m} \alpha_{k}\left(c_{i}\left(1-c_{r}\right)\right) Y_{k}^{(0)}\right) \\
&+ h^{2} \sum_{r=1}^{i} w_{r} k\left(t_{n}+c_{i} h, c_{r} h\right) \alpha_{j}\left(c_{i}-c_{r}\right)\left(\hat{y}_{0}+h \sum_{k=1}^{m} \alpha_{k}\left(c_{r}\right) Y_{k}^{(0)}\right), \\
& B_{i}^{(n)}=p\left(t_{n}+c_{i} h\right)+h \sum_{r=1}^{m} w_{i r} k\left(t_{n}+c_{i} h, t_{n}+c_{i} c_{r} h\right) \\
& \cdot\left(\hat{y}_{0}+h \sum_{k=1}^{m} \alpha_{k}\left(c_{i}\left(1-c_{r}\right)\right) Y_{k}^{(0)}\right) \\
&+h \sum_{r=1}^{i} w_{r} k\left(t_{n}+c_{i} h, c_{r} h\right)\left(\hat{y}_{0}+h \sum_{k=1}^{m} \alpha_{k}\left(c_{r}\right) Y_{k}^{(0)}\right)
\end{aligned}
$$




$$
\begin{aligned}
& C_{i}^{(n)}=q\left(t_{n}+c_{i} h\right)-h \sum_{k=1}^{n-1} \sum_{r=1}^{i} w_{r} k\left(t_{n}+c_{i} h, t_{k}+c_{r} h\right) \\
& \cdot\left(\hat{y}_{k}+h \sum_{j=1}^{m} \alpha_{j}\left(c_{r}\right) Y_{j}^{(k)}\right) \\
& \cdot\left(\hat{y}_{n-k}+h \sum_{j=1}^{m} \alpha_{j}\left(c_{i}-c_{r}\right) Y_{j}^{(n-k)}\right) \\
&-h \sum_{k=0}^{n-1} \sum_{r=i+1}^{m} w_{r} k\left(t_{n}+c_{i} h, t_{k}+c_{r} h\right)\left(\hat{y}_{k}+h \sum_{j=1}^{m} \alpha_{j}\left(c_{r}\right) Y_{j}^{(k)}\right) \\
& \cdot\left(\hat{y}_{n-k-1}+h \sum_{j=1}^{m} \alpha_{j}\left(1+c_{i}-c_{r}\right) Y_{j}^{(n-k-1)}\right) .
\end{aligned}
$$

We also have

$$
\hat{y}_{n}=\hat{y}_{n-1}\left(t_{n-1}+h\right)=\hat{y}_{n-1}+h \sum_{j=1}^{m} \alpha_{j}(1) Y_{j}^{(n-1)}, \quad n=1, \ldots, N .
$$

Note that $(3.5)$ is a nonlinear system. Fortunately, we can compute $Y_{i}^{(n)}$ and $\hat{y}_{n}(n \geq 1, i=1, \ldots, m)$ by (3.6) and (3.7) by solving systems of linear equations if we know the initial value $Y_{i}^{(0)}, i=1, \ldots, m$. Simple iteration, or Newton's iteration can be employed to determine the initial value from (3.5). It can be shown that for arbitrary given starting values $Y_{i}^{(0), 0}, i=1, \ldots, m$, the simple iteration method converges whenever $h>0$ is sufficiently small. We usually choose $Y_{i}^{(0), 0}=0, i=1, \ldots, m$; then Newton's iteration converges whenever $h>0$ is sufficiently small.

The implicit Runge-Kutta method (3.5)-(3.7) for (1.1) is characterized by the following arrays: the collocation parameters $\left\{c_{i} ; i=1, \ldots, m\right\}$ and the Gauss quadrature weights $\left\{w_{i j} ; i, j=1, \ldots, m\right\},\left\{w_{j} ; j=1, \ldots, m\right\}$. We choose them appropriately so that the results Theorem 3.1 and Corollary 3.1 of [4] are valid: $m=2, c_{1}=(3-\sqrt{3}) / 6, c_{2}=(3+\sqrt{3}) / 6, w_{1}=w_{2}=1 / 2$, and

$$
\begin{gathered}
W=\left(w_{i j}\right)=\left(\begin{array}{cc}
(3-\sqrt{3}) / 12 & (3-\sqrt{3}) / 12 \\
(3+\sqrt{3}) / 12 & (3+\sqrt{3}) / 12
\end{array}\right), \\
\left(\alpha_{1}(\tau), \alpha_{2}(\tau)\right)=\left(\tau\left(\tau-2 c_{2}\right) /\left[2\left(c_{2}-c_{1}\right)\right], \tau\left(\tau-2 c_{1}\right) /\left[2\left(c_{2}-c_{1}\right)\right]\right) .
\end{gathered}
$$

We consider the following examples.

Example 1.

$$
\begin{aligned}
& u^{\prime}(t)+\frac{1}{8} e^{-2 t} u(t)+\int_{0}^{t} \frac{1}{2} e^{-(t+s)} u(t-s) u(s) d s=-\frac{1}{4} e^{-t}+\frac{1}{32} e^{-2 t}, \quad 0 \leq t \leq 4, \\
& u(0)=\frac{1}{4} .
\end{aligned}
$$

The exact solution is $u(t)=\frac{1}{4} \cdot e^{-t}$. 
Example 2.

$$
\begin{aligned}
& u^{\prime}(t)+u(t)-\frac{3}{5} \int_{0}^{t} u(t-s) u(s) d s=30 \cdot(1+t) \cdot \cos (3 t), \quad 0 \leq t \leq 4 \\
& u(0)=0
\end{aligned}
$$

The exact solution is $u(t)=10 \cdot \sin (3 t)$.

There are some difficulties in obtaining accurate approximations in Example 2 , since the derivative of the exact solution changes rapidly on the interval $[0,4]$; see the results in Table II.

Example 3.

$$
\begin{aligned}
& u^{\prime}(t)+u(t)+\int_{0}^{t} t s u(t-s) u(s) d s=\frac{t^{5}}{60} \cdot\left(t^{2}-10 t+20\right)+t^{2}-2, \quad 0 \leq t \leq 4, \\
& u(0)=0 .
\end{aligned}
$$

The exact solution is $u(t)=t(t-2)$.

We list in Tables I, II, and III the resulting errors. By error we mean

$$
\text { error }=\mid \text { Exact value }- \text { Approximate value } \mid \text {. }
$$

The examples were solved on the HONEYWELL DPS8 in double precision. It appears that the implicit Runge-Kutta method we used has two major advantages: stability and accuracy. The main drawback is that the algorithms we used above are a little complicated compared with the multistep method presented in [8].

TABLE I

Errors for Example 1

\begin{tabular}{cccc}
\hline$t$ & $h=0.1$ & $h=0.05$ & $h=0.025$ \\
\hline 0.5 & $4.04 \times 10^{-9}$ & $2.19 \times 10^{-10}$ & $3.97 \times 10^{-12}$ \\
1.0 & $4.18 \times 10^{-9}$ & $2.12 \times 10^{-10}$ & $7.45 \times 10^{-12}$ \\
2.0 & $3.31 \times 10^{-9}$ & $1.64 \times 10^{-10}$ & $1.17 \times 10^{-11}$ \\
3.0 & $2.81 \times 10^{-9}$ & $1.34 \times 10^{-10}$ & $1.36 \times 10^{-11}$ \\
4.0 & $2.64 \times 10^{-9}$ & $1.23 \times 10^{-10}$ & $1.43 \times 10^{-11}$ \\
\hline
\end{tabular}

TABLE II

Errors for Example 2

\begin{tabular}{cccc}
\hline$t$ & $h=0.1$ & $h=0.05$ & $h=0.025$ \\
\hline 0.5 & $3.99 \times 10^{-5}$ & $2.36 \times 10^{-6}$ & $1.44 \times 10^{-7}$ \\
1.0 & $6.85 \times 10^{-5}$ & $4.31 \times 10^{-6}$ & $2.69 \times 10^{-7}$ \\
2.0 & $7.24 \times 10^{-4}$ & $4.54 \times 10^{-5}$ & $2.84 \times 10^{-6}$ \\
3.0 & $4.29 \times 10^{-3}$ & $2.68 \times 10^{-4}$ & $1.68 \times 10^{-5}$ \\
4.0 & $2.84 \times 10^{-2}$ & $1.79 \times 10^{-3}$ & $1.12 \times 10^{-4}$ \\
\hline
\end{tabular}


TABLE III

Errors for Example 3

\begin{tabular}{cccc}
\hline$t$ & $h=0.1$ & $h=0.05$ & $h=0.025$ \\
\hline 0.5 & $1.99 \times 10^{-9}$ & $1.84 \times 10^{-10}$ & $1.39 \times 10^{-11}$ \\
1.0 & $4.31 \times 10^{-8}$ & $3.17 \times 10^{-9}$ & $2.20 \times 10^{-10}$ \\
2.0 & $7.87 \times 10^{-7}$ & $5.32 \times 10^{-8}$ & $3.50 \times 10^{-9}$ \\
3.0 & $6.74 \times 10^{-6}$ & $4.47 \times 10^{-7}$ & $2.87 \times 10^{-8}$ \\
4.0 & $7.00 \times 10^{-5}$ & $4.62 \times 10^{-6}$ & $2.94 \times 10^{-7}$ \\
\hline
\end{tabular}

\section{BIBLIOGRAPHY}

1. M. Aguilar and H. Brunner, Collocation methods for second-order Volterra integro-differential equations, Appl. Numer. Math. 4 (1988), 455-470.

2. C. T. H. Baker, Initial value problems for Volterra integro-differential equations, in Modern Numerical Methods for Ordinary Differential Equations (G. Hall and J. M. Watt, eds.), Clarendon Press, Oxford, 1976, pp. 296-307.

3. H. Brunner, On the numerical solution of nonlinear Volterra integro-differential equations, BIT 13 (1973), 381-390.

4. __ Implicit Runge-Kutta methods of optimal order for Volterra integro-differential equations, Math. Comp. 42 (1984), 95-109.

5. H. Brunner and J. D. Lambert, Stability of numerical methods for Volterra integro-differential equations, Computing 12 (1974), 75-89.

6. H. Brunner and P. J. van der Houwen, The numerical solution of Volterra equations, CWI Monographs, vol. 3, North-Holland, Amsterdam and New York, 1986.

7. LI. G. Chambers, Integral equations: A short course, International Textbook Co., London, 1976.

8. S. H. Chang and J. T. Day, On the numerical solution of certain nonlinear integro-differential equations, J. Comput. Phys. 26 (1978), 162-168.

9. C. W. Cryer, Numerical methods for functional differential equations, in Delay and Functional Differential Equations and Their Applications (K. Schmitt, ed.), Academic Press, New York, 1972, pp. 17-101.

10. J. A. Dixon, A nonlinear weakly singular Volterra integro-differential equation arising from a reaction-diffiusion study in a small cell, J. Comput. Appl. Math. 18 (1987), 289-305.

11. C. M. Elliott and S. McKee, On the numerical solution of an integro-differential equation arising from wave-power hydrolics, BIT 21 (1981), 318-325.

12. A. Feldstein and J. R. Sopka, Numerical methods for nonlinear Volterra integro-differential equations, SIAM J. Numer. Anal. 11 (1974), 826-846.

13. C. Lubich, Diploma Thesis, University of Innsbruck, 1981.

14. __ Runge-Kutta theory, for V'olterra integro-differential equations, Preprint No. 131, Sonderforschungsbereich 123, University of Heidelberg, 1981.

15. A. Makroglou, Convergence of a block-by-block method for nonlinear Volterra integro-differential equations, Math. Comp. 35 (1980), 783-796.

16. S. McKee, The analysis of a variable step, variable coefficient linear multistep method for solving a singular integro-differential equation arising from the diffusion of discrete particles in a turbulent fluid, J. Inst. Math. Appl. 23 (1979), 373-388.

17. W. L. Mocarsky, Convergence of step-by-step methods for nonlinear integro-differential equations, J. Inst. Math. Appl. 8 (1971), 235-239.

18. A. S. Monin and A. M. Yaglom, Statistical hydromechanics, Part 2, "Nauka", Moscow, 1967. (Russian) 
19. A. Prosperetti, A numerical method for the solution of certain classes of nonlinear Volterra integro-differential and integral equations, J. Numer. Methods Engrg. 11 (1977), 431-438.

20. T. L. Saaty, Modern nonlinear equations, McGraw-Hill, New York, 1967.

21. Tang Tao and Yuan Wei, The further study of a certain nonlinear integro-differential equation, J. Comput. Phys. 72 (1987), 486-497.

22. B. A. Velikson, Solution of a nonlinear integro-differential equation, U.S.S.R. Comput. Math. and Math. Phys. 15 (1975), 256-259.

23. V. Volterra, Theory of functionals and of integral and integro-differential equations, Dover, New York, 1959.

Department of Mathematics, Peking University, Beijing, China 